
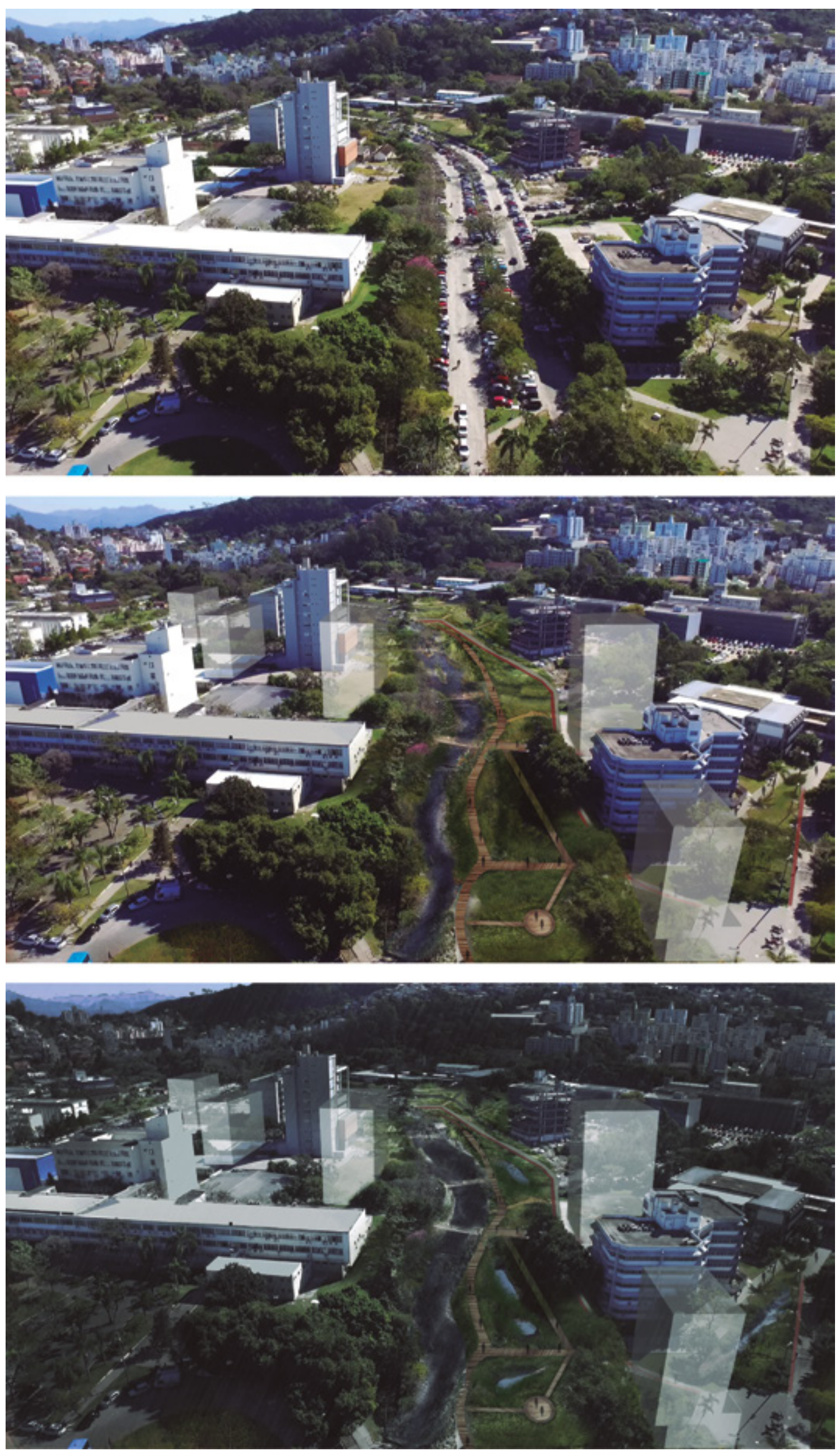

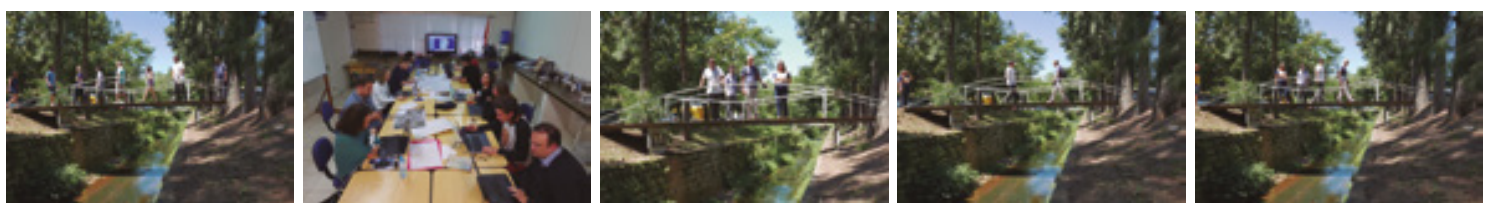

Secuencia: Paseo por el campus y con alumnos en el aula

Fotos: José Kós e Maria Paula

\title{
PROYECTO REGENERATIVO DEL CAMPUS: APRENDIENDO A TRAVÉS DE LAS DINÁMICAS DE LA NATURALEZA Y LA UNIVERSIDAD ${ }^{1}$
}

\author{
REGENERATIVE CAMPUS DESIGN: LEARNING FROM NATURE AND UNIVERSITY \\ DYNAMICS 1
}

\author{
José Ripper Kós², Marila Filartiga Gebara³, Cesar Augusto Pompêo ${ }^{4}$, Luís Henrique \\ Pavan $^{5}$
}

RESUMEN

\begin{abstract}
La sustentabilidad del planeta exige un cambio significativo en la forma cómo nos relacionamos con el medio ambiente. En ese contexto, el gran desafío consiste en pasar del discurso a la práctica. Las universidades, como formadoras de futuros profesionales e investigadores, deberían asumir una posición de liderazgo en este proceso. Sin embargo, la fragmentación del conocimiento, en áreas o departamentos aislados, no contribuye a la implementación de esos cambios relevantes. El proceso de desarrollo de un proyecto regenerativo para el campus de la Universidad Federal de Santa Catarina, en Brasil, constituye una propuesta para el debate sobre el papel del espacio físico universitario como instrumento pedagógico para la resiliencia de los ecosistemas. El concepto del proyecto surge del análisis y las proposiciones de estudiantes de posgrado, provenientes de diferentes áreas formativas, las cuales se orientan a desarrollar propuestas claras en las que dinámicas y ciclos de la naturaleza puedan aportar positivamente a la calidad de aprendizaje y viceversa.
\end{abstract}

Palabras clave: arquitectura ambiental, desarrollo sostenible, campus universitario, educación universitaria, hidrología.

\section{ABSTRACT}

The sustainability of the planet requires a significant change in the way we relate to the environment. In this context, the great challenge is to move from discourse to practice. Universities, which are responsible for educating future professionals and researchers, should take a leadership position in this process. However, the fragmentation of knowledge into isolated areas or departments does not contribute to the implementation of these relevant changes. The process of developing a regenerative project for the main campus of Santa Catarina University in Brazil relates directly to the debate on the role of the physical space of universities as a pedagogical instrument for the resilience of ecosystems. The design concept arose from the analyses and propositions of graduate students from different disciplines. They aim to develop clear proposals in which nature's dynamics and cycles positively contribute to the quality of learning and vice versa.

Keywords: environmental architecture, sustainable development, university campus, university education, hydrology.

Artículo recibido el 15 de agosto de 2017 y aceptado el 1 de diciembre de 2017 DOI: https://doi.org/10.22320/07196466.2017.35.052.04

[1] Este trabajo está inserto en el Proyecto CITYLABS, con recursos del Programa Erasmus + de la Comunidad Europea. También fue financiado por la Universidade Federal de Santa Catarina y el Conselho Nacional de Desenvolvimento Científico e Tecnológico (CNPq). [2] Departamento de Arquitetura e Urbanismo - Universidade Federal de Santa Catarina, Florianápolis, Brasil. jose.kos@ufsc.br

[3] Programa de Pós-Graduação em Arquitetura e Urbanismo - Universidade Federal de Santa Catarina, Florianápolis, Brasil. m.filartiga@posgrad.ufsc.br

[4] Departamento de Engenharia Sanitária e Ambiental - Universidade Federal de Santa Catarina, Florianápolis, Brasil. cesar. pompeo@ufsc.br

[5] Laboratório de Ecologia Urbana - Universidade Federal de Santa Catarina, Florianápolis, Brasil. Iuis.henrique.pavan@grad.ufsc.br 
La necesidad de alterar, de forma relevante, nuestras actitudes en relación al medio ambiente es casi unánime. Sin embargo, el transitar del discurso a la práctica en este tema es extremadamente difícil, por lo que aún no han sido implementados cambios significativos. Uno de los argumentos, en este sentido, es la dificultad de crear de soluciones efectivas a problemas, a través de la misma lógica que los originó. Además de eso, no podemos olvidar que, en las grandes ciudades, consumimos productos traídos de diversas partes del planeta y vivimos la naturaleza sintetizada a través de una colección de mercaderías o en lugares de recreación, que crean una fuerte abstracción de nuestra relación con la naturaleza. Esta abstracción nos aleja de la noción de que no estamos simplemente conectados con la naturaleza, somos naturaleza, y ella es la real fuente de nuestras vidas y de nuestro bienestar (Wackernagel y Rees, 1996).

De acuerdo con Wu y Wu (2013: 212), "una miríada de factores es responsable por el actual estado insustentable del mundo. Dos de ellos son particularmente importantes de ser mencionados: nuestra comprensión inadecuada o incorrecta de como la naturaleza funciona en la ciencia e nuestro inadecuado o mal uso del conocimiento ecológico en acción. Nuestra percepción de naturaleza ha sido modelada por mitos y creencias, como el equilibrio de la naturaleza que viene siendo una importante base de fondo en ecología". Recurriendo a diversas lecturas, estos autores demuestran la importancia de comprender las dinámicas de la naturaleza y cómo ellas están lejos de nuestra creencia de que son sistemas ordenados o en un estado de constante equilibrio.

Las universidades, responsables por la formación de gran parte de los profesionales capaces de promover estos cambios, deberían liderar este proceso. Pero estas continúan rigiéndose por modelos y estructuras que estimulan la fragmentación del conocimiento, aún después del reconocimiento de la necesidad de reintegración de conocimientos y saberes con la publicación "Our Common Future" (Brundtland, 1987) y la Agenda XXI de los años 90. Recientemente, algunas universidades vislumbraron la posibilidad de revisión de esta fragmentación a través de profundas alteraciones en sus espacios físicos. Una de las principales iniciativas, en curso actualmente, es el nuevo campus de Cornell Tech en Manhattan, que explora oportunidades de encuentros informales, o de interacción incidentales, consideradas por muchos como una de las principales contribuciones del campus contemporáneo para modelos innovadores de aprendizaje, que no pueden ser replicados en línea de forma eficaz. El nuevo campus fue defendido por su presidente Elizabeth Garrett, a través de un discurso en esa dirección: “Debemos trabajar juntos para entender los difíciles problemas de nuestra era -entre ellos, la sustentabilidad y los cambios climáticos; nuevos abordajes para la salud y el bienestar; el reto de la creciente desigualdad global y doméstica; la influencia de la tecnología; y el proyecto de instituciones democráticas efectivas- y elaborar soluciones a través de colaboración interdisciplinar e intercampus" 
(2015: 7). Este debate viene ganando fuerza e iniciativas iguales son discutidas en propuestas para los campus del M.I.T., Columbia, y otras reconocidas universidades. Ahora bien, ya en el inicio de los año 60, Buckminster Fuller criticaba la forma como la sociedad asumía "natural, inevitable y deseablemente" la creciente especialización de la estructura universitaria. En contra de la idea de que los comportamientos de los sistemas podían ser previstos por el estudio separado de sus partes, acudió a la palabra "sinergia" (synergy), la única en la lengua inglesa que afirma precisamente que "el comportamiento de sistemas enteros no puede predecirse a partir de los comportamientos observados separadamente de cualquiera de las partes del sistema o cualquier sub-montaje de las partes del sistema" (1969: 78).

La estructura académica basada en especializaciones fue formalizada en los espacios físicos de la mayoría de las universidades. Además de estudiar el medio ambiente de manera fragmentada, la inserción del campus universitario en los diferentes ecosistemas confirma este concepto, una vez que la comunidad universitaria vive diariamente esa fragmentación en su espacio de aprendizaje. Inclusive en detalles, como en la solución de problemas comunes de mantenimiento, reestructuraciones puntuales de los espacios libres o en la manera de operar los edificios académicos, difícilmente son ofrecidos buenos ejemplos de cómo pensar el ambiente universitario como parte de un ecosistema o de un sistema integral.

Desde esa perspectiva, Orr plantea que "el problema no es solo que muchos edificios académicos son antiestéticos, no funcionan muy bien o no encajan en su lugar o región. El problema más profundo es que los edificios académicos no son factores neutros y asépticos en el proceso de aprendizaje. Asumimos, equivocadamente, que el aprendizaje ocurre en edificios, pero ignoramos la influencia del proyecto, su posición en el terreno, de los materiales constructivos y de cómo estos edificios operan en el aprendizaje de sus ocupantes. El punto es que la arquitectura académica posee una especie de pedagogía cristalizada y los edificios tienen su propio currículo oculto que enseña tan efectivamente como cualquier curso enseñado en ellos". [...] "Se sigue que los aspectos éticos, ecológicos o estéticos de la construcción no importan tanto como la técnica y la tecnología" (2004:112-113).

La forma cómo los espacios físicos de las universidades son estructurados y administrados no ofrece a la comunidad académica, y a la sociedad en general, buenos ejemplos de integración con el medio ambiente. La fragmentación de dicha relación es evidenciada en las actitudes para resolver problemas comunes, por ejemplo, el modo en que los locales de aprendizaje son organizados a través del campus. El campus de la mayoría de las grandes universidades con amplia extensión territorial posee, sin duda, un importante potencial para promover formas innovadoras de relación con el medio ambiente. No obstante, ello raramente sucede: las universidades desperdician una excelente oportunidad de demostrar que sus estudiantes forman, efectivamente, parte de un medio ambiente en constante proceso de regeneración e, incluso, que la dinámica de la universidad puede mejorar las condiciones ambientales generalmente degradadas por su entorno urbano. Los campus universitarios sugieren que las actividades académicas no son directamente compatibles con el desarrollo adecuado de la fauna, flora, cursos de agua, suelo, etc. Además, estos temas son tratados de forma aislada dejando de considerarlos como parte de un sistema único. Y, aunque gran parte de los estudiantes aborde en el currículo de sus carreras cuestiones relacionadas al medio ambiente, estas son tratadas solo a partir de sus áreas de conocimiento, sin una mirada que estimule la construcción de una ética ambiental en el cotidiano, la que estaría reservada únicamente al ejercicio profesional.

¿Cómo esperar que universidades asentadas en estructuras académicas fragmentadas, que no se consideran como parte integrante del medio ambiente, sino como algo separado, puedan formar una generación con la responsabilidad de promover los cambios necesarios para una nueva forma de habitar el planeta?

A través del estudio del origen de la palabra "cura" (heal), Bill Reed revela el concepto de "crear el todo" (to make whole). Sugiere que "cuando substituimos nuestro modelo mental de un proceso de interrupción de deterioración por otro en que los seres humanos son parte integrante de la naturaleza, también cambiamos nuestra conciencia. Cuando comenzamos a participar de la naturaleza, descubrimos que los seres humanos que trabajan de forma para curar la naturaleza, en última instancia, también se curarán y desarrollarán. Esa transformación no sucede de un día para otro, mas $\mathrm{s}$ a través de un proceso continuo de cura o de creación de todo" (7group y Reed, 2009: 378). La mayoría de las huertas domésticas, en el medio rural, son cuidadas por las mujeres. A ellas se las relaciona otro término, "care", que también tiene que ver con el cuidado materno. Comúnmente, se menciona a la madre tierra, "pacha mama", de la cual los seres vivos se nutren.

Las principales universidades, en su búsqueda por procesos innovadores y creativos en investigación y aprendizaje, estimulan la colaboración y la transdisciplinaridad. Los problemas complejos con los cuales nuestras sociedades frecuentemente se encuentran no pueden ser abordados a través de una estructura tradicional, fragmentada en diferentes áreas del conocimiento. Es necesario substituir un pensamiento que aísla y separa, por otro que distingue y une. El pensamiento disyuntivo y reductor debe dar lugar a un pensamiento complejo, en el sentido originario del término complexus: lo que es tejido junto (Morin, 1994). Este pensamiento es necesario para la clara comprensión de las dinámicas de los ecosistemas, a lo largo de la historia.

Si bien es cierto que, cuando las universidades brasileñas se encuentran imbuidas de preocupaciones sustentables, no huyen del modelo reducción de los impactos negativos causados al medio ambiente, la verdad es que desperdician la posibilidad de crear un impacto ambiental positivo para regenerar situaciones de degradaciones. McDonough y Braungart (2002:45) destacan, en este sentido, que "ser menos malo no es ser bueno". 


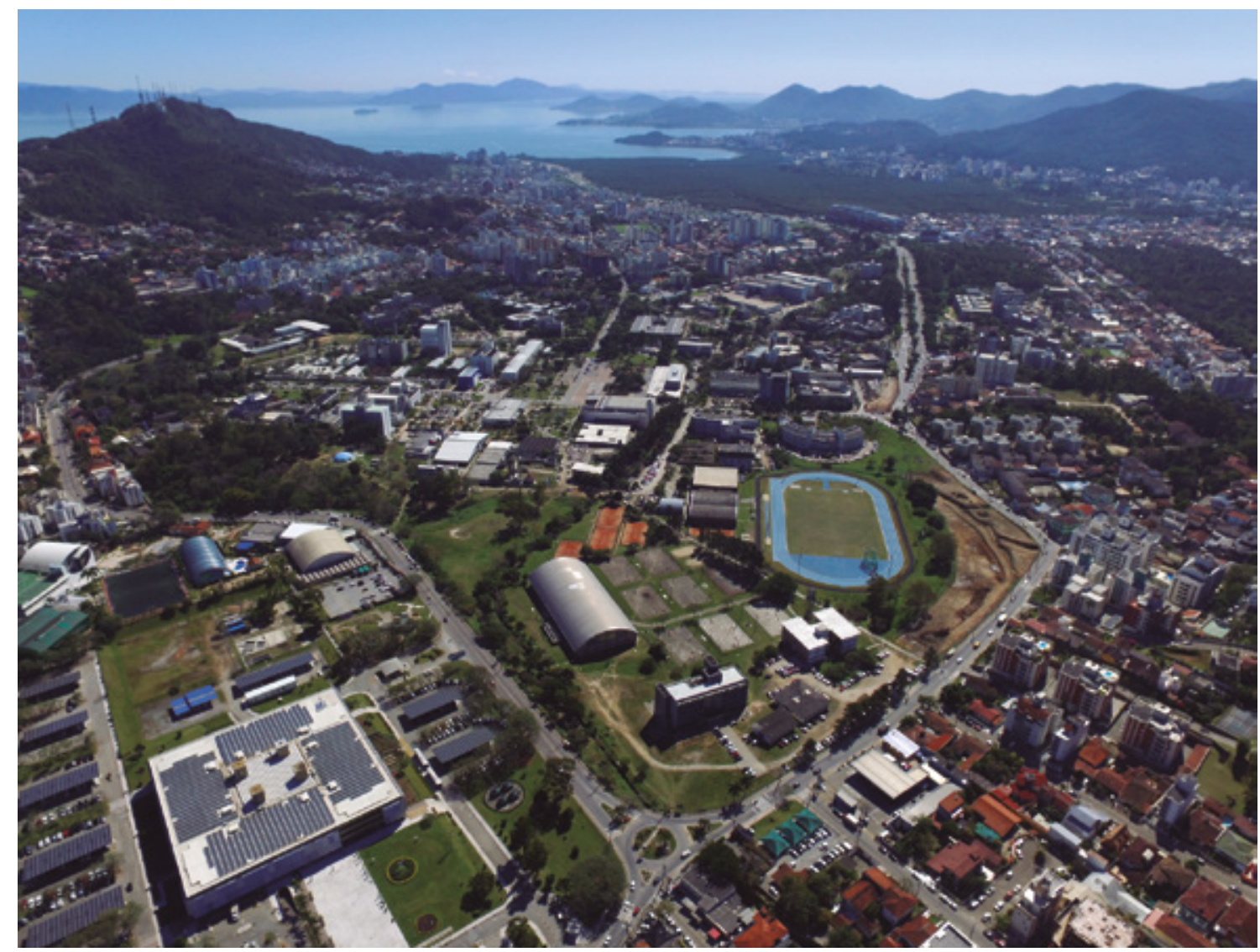

Figura 1 Campus de la Universidade Federal de Santa Catarina. Fuente: Laboratório de Ecologia Urbana (UFSC). Fotografía de Felipe Finger

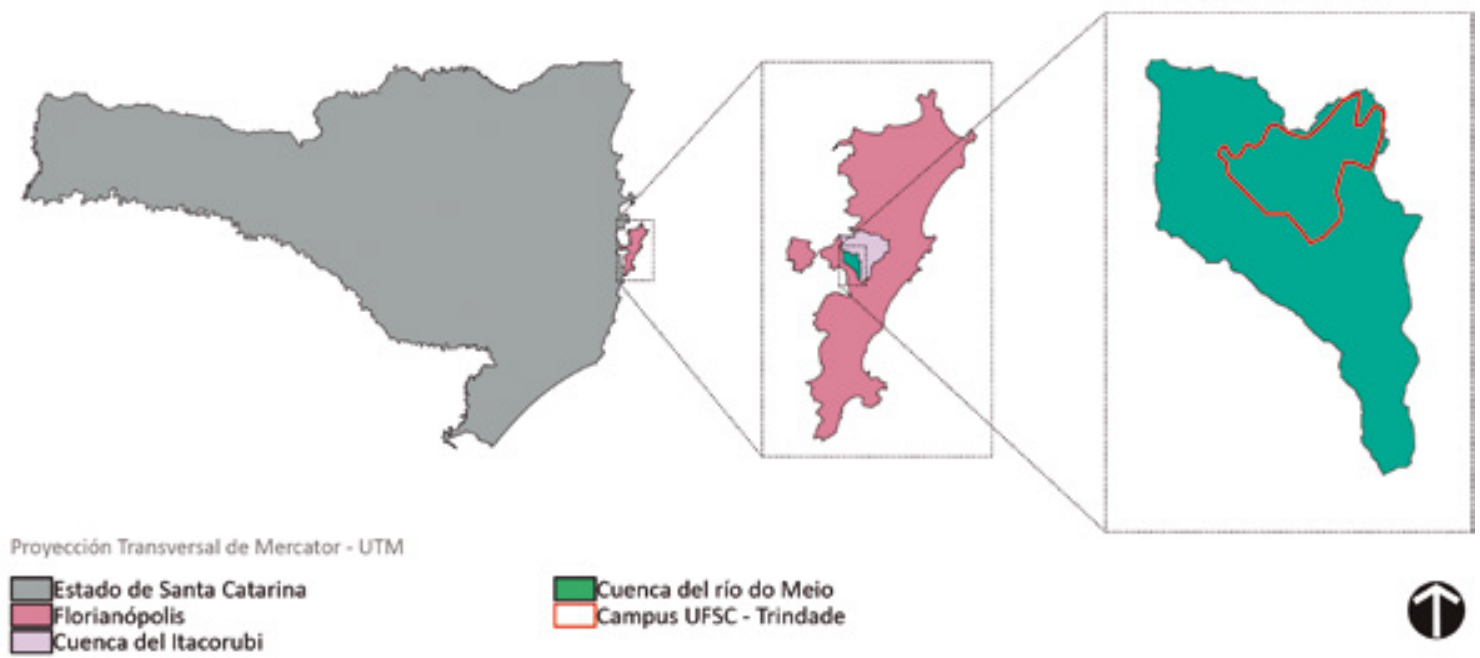

Figura 2 Ubicación del campus de la Universidad Federal de Santa Catarina. Fuente: Núcleo de Estudos da Água (UFSC) 
potencial de promoción de valorización de las áreas vecinas, recibiría la parte más delicada de la región. Por

Desde 2016, estos temas constituyen la base de una investigación relacionada con el principal campus de la Universidade Federal de Santa Catarina (UFSC) (Figura 1), en forma de una experiencia que propone la renovación de formatos de aprendizaje para toda la comunidad académica. Estas experiencias toman como punto de partida la universidad como parte de un ecosistema delicado e importante para todas las redes naturales de la región. Un proyecto con este abordaje debe constituir una oportunidad de promoción de cambios estructurales en la manera en que una comunidad se percibe como parte de este ecosistema. Por esta razón, este proyecto es estudiado por su potencial de ser un agente impulsor de un nuevo contexto para el ecosistema de la región que busca revertir el proceso de degradación ocurrido a lo largo del tiempo y, en definitiva, promover un proceso de regeneración ambiental.

Es importante considerar que "Currently, the predominant narrative of sustainability is that of scarcity, negative impacts and disruptive change in the face of growing socioeconomic needs. The subtext is that of uncertainty and sacrifice, which in turn engenders resistance to change". (Hes y du Plessis, 2015:12-13). Así, en vez de buscar reducir los efectos negativos del campus en el medio ambiente, el proyecto persigue generar un impacto positivo en términos ambientales, pero también apunta a una revisión del proceso de aprendizaje de la propia universidad. Bill Reed defiende, desde este enfoque, que "regeneración es sobre-proyectar para el todo: involucrar los sistemas de la tierra, los sistemas bióticos y las personas (o los sistemas humanos) de cada lugar específico, en un diálogo continuo para apoyar su desarrollo coevolutivo. Regenerar significa ofrecer a este lugar una nueva vida y una nueva energía. La vida y la energía sustentable solo pueden suceder en un sistema como un todo. Este no es un preciosismo intelectual -desarrollar relaciones entre los seres vivos que componen un sistema entero es necesario para alcanzar una condición verdaderamente saludable y sustentable" (7group y Reed, 2009: 46).

Entender los patrones que estructuran el ecosistema de la región donde se ubica el campus es esencial para un proceso cuidadoso de un proyecto regenerativo: "Cuando buscamos los patrones naturales que dieron forma a un lugar a lo largo del tiempo, nuevas posibilidades de desarrollo responsable son reveladas" (7group y Reed, 2009:58).

Para contextualizar el proyecto aquí expuesto, debe indicarse que la instalación del campus de la UFSC en el barrio Trindade, en Florianópolis, ocurrió en la década de los 60, luego de largos debates. El área cedida era extremadamente interesante por su gran cantidad de tierras. Aunque extensa, dicha área destinada a la universidad, siguió una lógica común en donaciones de esta naturaleza. La universidad pública, con gran esta razón, se discutió mucho la viabilidad de su instalación en un área distante e inundable. Esta región, cercada de cerros y con pequeño desnivel en relación al mar, recogía las aguas de los cerros circundantes, direccionándolas hacia una región de manglares. La dinámica de las aguas del nuevo terreno de la universidad siempre fue encarada como un problema, un problema constantemente esgrimido por los opositores del traslado de la universidad del área central de la ciudad. En aquella época, no fue realizado ningún estudio para integrar esta dinámica al funcionamiento de la universidad. La estrategia adoptada, que no es muy diferente de la adoptada en situaciones semejantes actualmente, fue construir una red de drenaje, canalizando las aguas. Para esto, fueron realizadas obras caras, principalmente si consideramos los recursos disponibles para la nueva universidad, las cuales se dificultaron aún más por las constantes inundaciones que sufrió la zona (Rodrigues, 2010). Estos canales rectificados buscaban drenar rápidamente las aguas en la dirección hacia el manglar y, posteriormente, hacia el mar. El desarrollo de los terrenos en el entorno de la ciudad ya era previsto, pero fue poco considerado en el proyecto de drenaje. Con eso, la solución técnica resolvió parcialmente el problema de retirada de aguas del terreno, pero dejó a la universidad más vulnerable. En épocas de muchas lluvias -y especialmente cuando estas vienen acompañadas de un régimen de mareas muy altas-, estos canales no son suficientes para recoger el volumen de aguas generado debido a la forma en que las áreas del entorno fueron urbanizadas. El aumento de la velocidad de las aguas pasa a ser una de las causas de las serias y constantes inundaciones en el campus.

El Campus Universidad Federal de Santa Catarina se encuentra situado en la Subcuenca del Río do Meio (o Río do Sertão), integrante de la Cuenca del Itacorubi, ubicada en la región centro-oeste de la Isla de Santa Catarina, ciudad de Florianópolis, capital del Estado de Santa Catarina, al sur de Brasil (Figura 2). La cuenca del rio do Meio posee un área de $4,5 \mathrm{~km}^{2}$, su cauce principal presenta $3 \mathrm{~km}$ de extensión, siendo cerca de $0,3 \mathrm{~m}^{3} / \mathrm{s}$ su caudal promedio en tiempos secos, sin lluvia en los días anteriores. El área del Campus alcanza 1,2km².

Una particularidad de la Cuenca del Itacorubi es su relación con tres Unidades de Conservación: el Parque Urbano del Morro da Cruz (PUMC), el Parque Municipal del Manguezal del Itacorubi (PMMI) y el Parque Municipal del Macizo de la Costera (PMMC). En términos de importancia para la conservación de los recursos hídricos y biodiversidad, el Manguezal del Itacorubi y el Macizo de la Costera ocupan posiciones destacadas. En ese sentido, los ríos do Meio y Córrego Grande poseen gran potencial de retornar a ser corredores ecológicos entre las dos Unidades de Conservación. Sus nacientes se sitúan en el Parque Municipal del Macizo de la Costera y la desembocadura de ambos, en el Parque Manguezal del Itacorubi (Figura 3). 


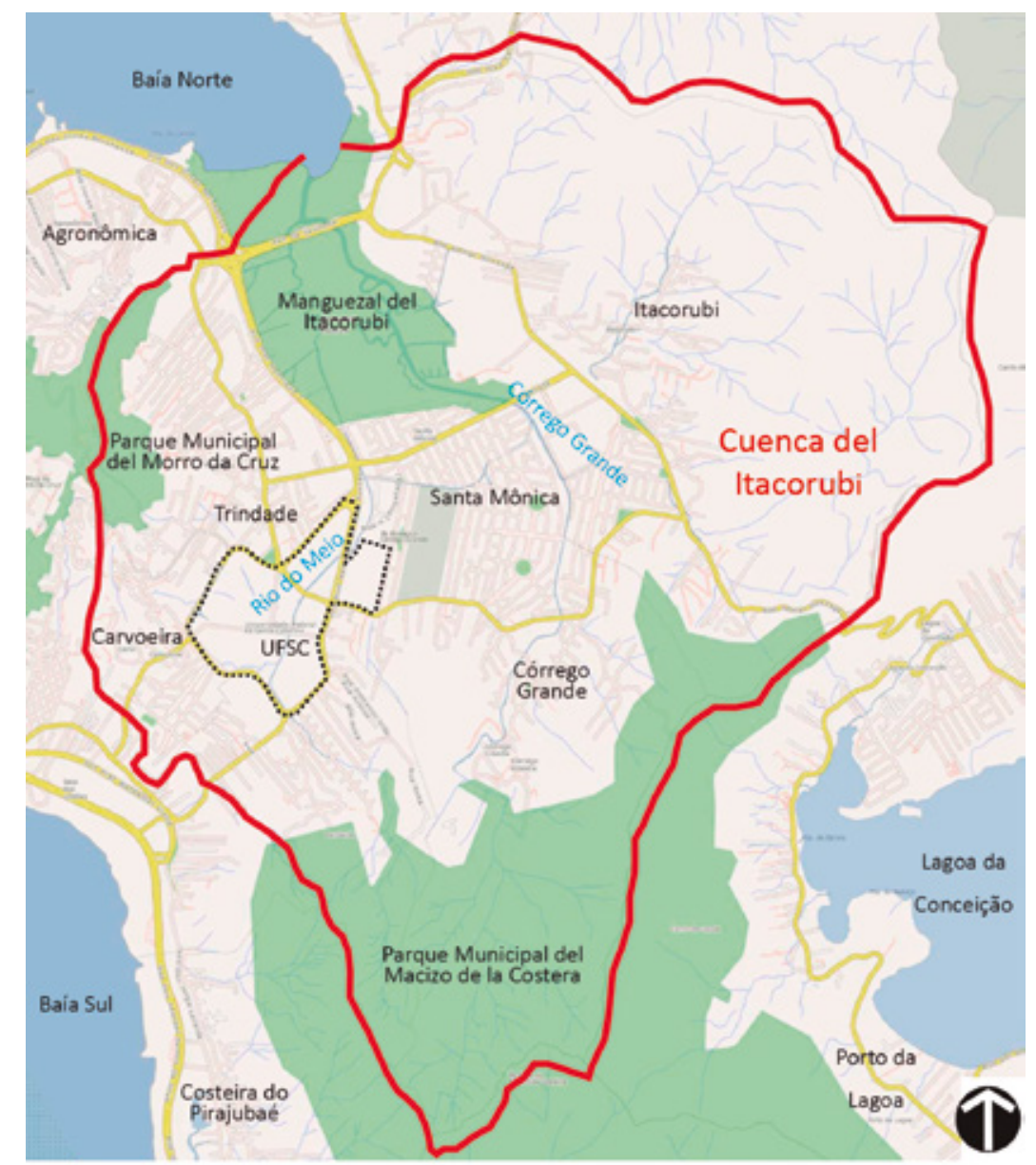

Cuenca del Itacorubi

Campus UFSC - Trindade

Figura 3 Unidades de Conservación en la Cuenca del Itacorubi. Fuente: Núcleo de Estudos da Água (UFSC) adaptado de PMF - Geoprocesamiento Corporativo (2017).

módulo 1 - 11

806

doctorado

80 maestria

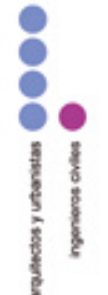

aspectos trabajados

1098

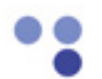

grado

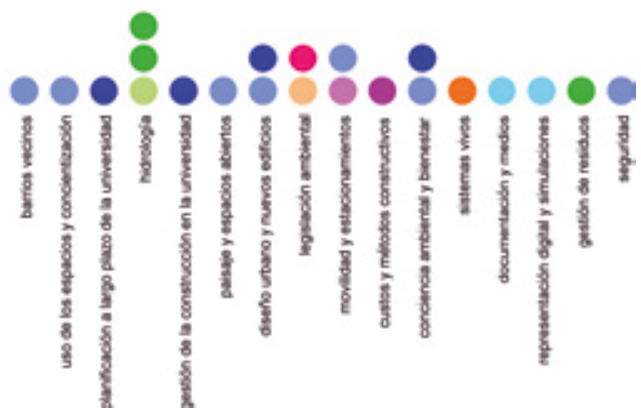

Figura 4 Composición de los módulos (junio - septiembre 2016 y septiembre - diciembre 2016). Fuente: Laboratório de Ecologia Urbana (UFSC). 
De esta forma, la universidad posee hoy una situación compleja, pero extremadamente rica para pensar su futuro. La forma como la UFSC se relaciona con los ecosistemas de la región ofrece una oportunidad para el estudio y el debate del papel de la universidad en un momento sensible en todo el planeta. La investigación descrita en este artículo está siendo estructurada por intermedio de disciplinas de posgrado junto al Programa de Pos-grado en Arquitectura y Urbanismo de la UFSC, destinadas a estimular el debate de diferentes puntos de vista sobre la relación de la universidad con los ecosistemas locales y la discusión de las posibilidades de interacción entre la universidad, la administración municipal y las comunidades vecinas, para la conservación y la recuperación de los ecosistemas.

Una de las maneras de propiciar que los futuros profesionales, de diferentes áreas, resulten preparados para promover alteraciones importantes en nuestras relaciones con el medio ambiente es a través de un pensamiento complejo, como el defendido por Morin (1990) y Leff (2001). Para ello, es importante abordar estas problemáticas con una visión más amplia. Si no, difícilmente se conseguirá formar profesionales como Leonardo Da Vinci, en las palabras de Buckminster Fuller (1969), el ejemplo excepcional de científico diseñador capaz de anticipar todo, o incluso como Buckminster Fuller. El creciente desarrollo de las áreas específicas del conocimiento prácticamente inviabiliza la formación de este perfil de profesionales. Si la especialización es una de las barreras para promover estos cambios estructurales, se debe reducir entonces la dificultad de comunicación entre profesionales de diferentes áreas. Choi y Park, a partir de una cuidadosa investigación sobre los términos "multidisciplinariedad", "interdisciplinariedad" y "transdisciplinariedad", declaran que "la yuxtaposición ambigua e incongruente de elementos de información heterogéneos que están relacionados a través de la operación de una interfaz transdisciplinar, probablemente estimulará el surgimiento de nuevos conocimientos" (2006: 357). Facilitar la comunicación explorando la diversidad de los integrantes del grupo es esencial para la construcción de esta interfaz transdisciplinar. La universidad puede, a través de colaboración y transdisciplinariedad, potencializar sus actividades, descubriendo nuevas maneras de alcanzar un pensamiento que distinga y, a la vez, una.

Por lo tanto, uno de los supuestos de esta investigación es que el proceso de proyecto para el nuevo campus debe integrar diferentes áreas del conocimiento, vislumbrando nuevas formas de aprendizaje para toda la universidad. La calidad del ecosistema urbano para la región estará conectada a la complejidad de la formulación de los problemas relacionados con él. La regeneración del ecosistema natural deberá estimular interacciones entre fauna, flora y las actividades realizadas en este nuevo ambiente. Cuanto más positiva sea esta interacción para todos, mayores serán las posibilidades de garantía de un ecosistema urbano resiliente que se desarrolle de forma natural. Una nueva universidad, con este abordaje, tendrá muchas más oportunidades de promover las alteraciones exigidas para el bienestar de nuestras futuras generaciones.

\section{RESULTADOS}

A comienzos de 2016, profesores de los Departamentos de Arquitectura y Urbanismo, Ingeniería Sanitaria y Ambiental e Ecología iniciaron la planificación de disciplinas en el Programa de Posgrado en Arquitectura y Urbanismo de la UFSC. Estas disciplinas debían ser lo suficientemente flexibles para acoger a estudiantes de diversas áreas del conocimiento. Al presente, ya fueron realizados dos módulos, cada uno en un trimestre. Concretamente, el objetivo de los módulos fue debatir una nueva propuesta de universidad a partir de la comprensión de su espacio físico y sus patrones, tanto de los existentes como de los anteriores que han sido modificados a los largo del tiempo, con miras a desarrollar un proyecto regenerativo para toda la región. El primer módulo contó con 11 estudiantes, cada uno de cuales asumió el papel de diferentes profesionales o de partes interesadas en el proyecto, dirigiendo sus estudios y contribuciones a diferentes áreas del conocimiento, y relacionando estos, siempre que fuera posible, con su área de investigación académica (Figura 4).

Considerando que el campus posee una malla hídrica que, aunque conduzca bajos caudales en periodos secos, presenta dimensiones capaces de acomodar mayores flujos en periodos lluviosos y, de esta manera, se constituye en un elemento definitorio del paisaje y de la movilidad humana en el interior del campus, el punto de partida de estos módulos fue el análisis de la dinámica de las aguas, especialmente sensible en esa región. Como se mencionó, durante el proceso de instalación del campus, los cursos de agua naturales sufrieron alteraciones del trazado y de la forma de su sección para un rápido flujo de las aguas provenientes de las áreas más altas de la cuenca del Río do Meio. Si bien estas intervenciones son bastante visibles (Figura 5), representaron una solución destinada a fracasar porque el proceso de urbanización de esas áreas elevó la magnitud de las descargas y redujo el tiempo de respuesta de la cuenca, concentrando el flujo justo en la región del campus universitario cuyo relieve es significativamente suave y también sufre influencia de mareas elevadas. En la actualidad, la universidad continúa considerando los cursos de agua como un problema que debe ser evitado. En consecuencia, los canales no son valorizados ni están incluidos en los espacios universitarios. Las posibilidades dadas por las características propias de la región que podrían representar un instrumento de aprendizaje son desperdiciadas, pasando a actuar de manera opuesta. Como muestra de ello, la comunidad académica considera los ciclos ambientales como algo externo a sus actividades y hasta potencialmente negativo. Asimismo, los márgenes de estos canales, que deberían ser priorizados en términos de protección, son en gran parte ocupados por grandes estacionamientos. Por ello, el estudio del flujo de carros, de los procesos de movilidad en la ciudad y de la manera cómo las personas se encuentran en la universidad constituyen algunos de los principales aspectos estudiados en esta iniciativa, junto con las características de los diversos canales en el campus. 


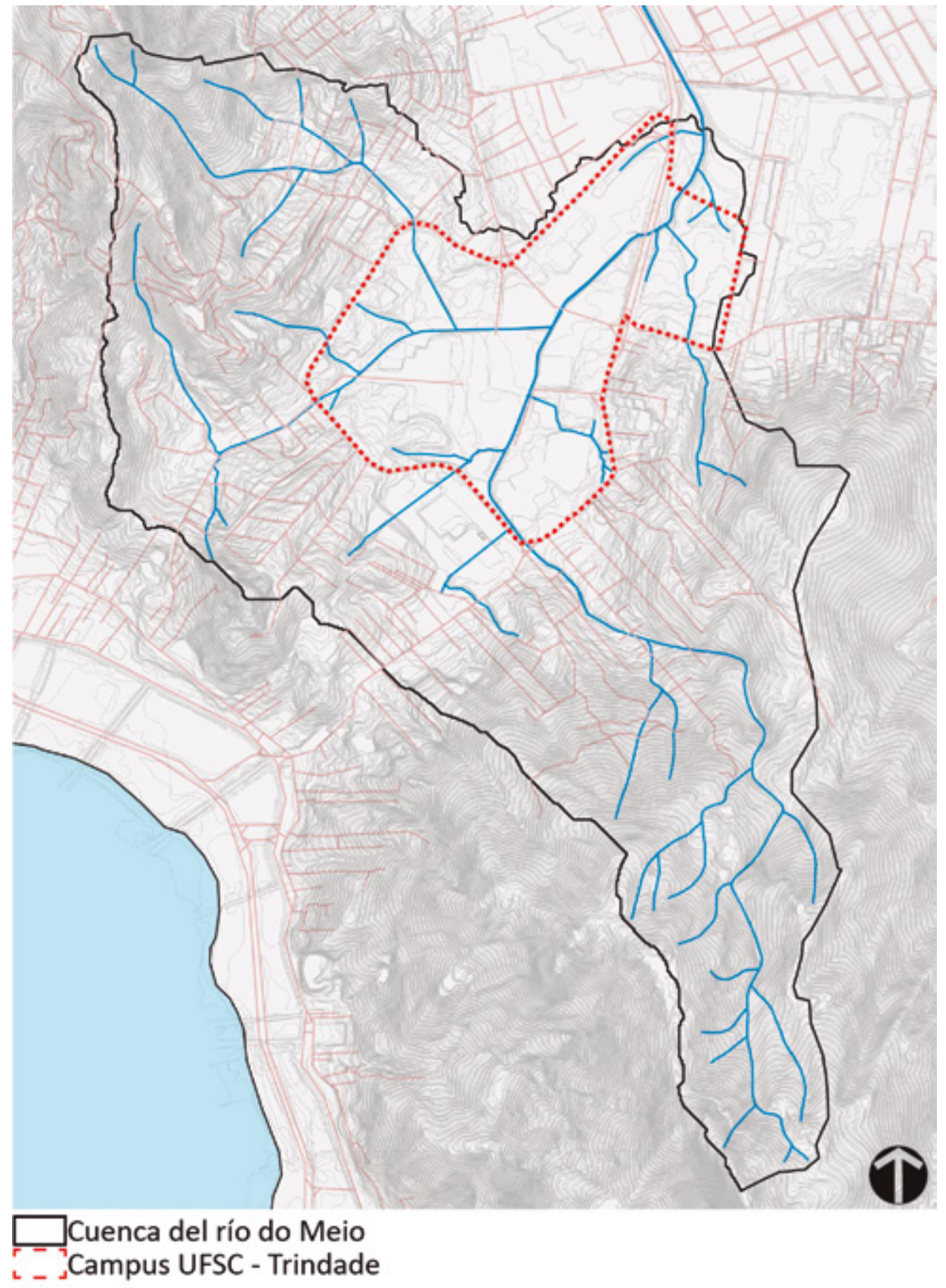

Figura 5 Hidrografía de la cuenca del Río do Meio y el campus de la UFSC. Fuente: Núcleo de Estudos da Água (UFSC) 

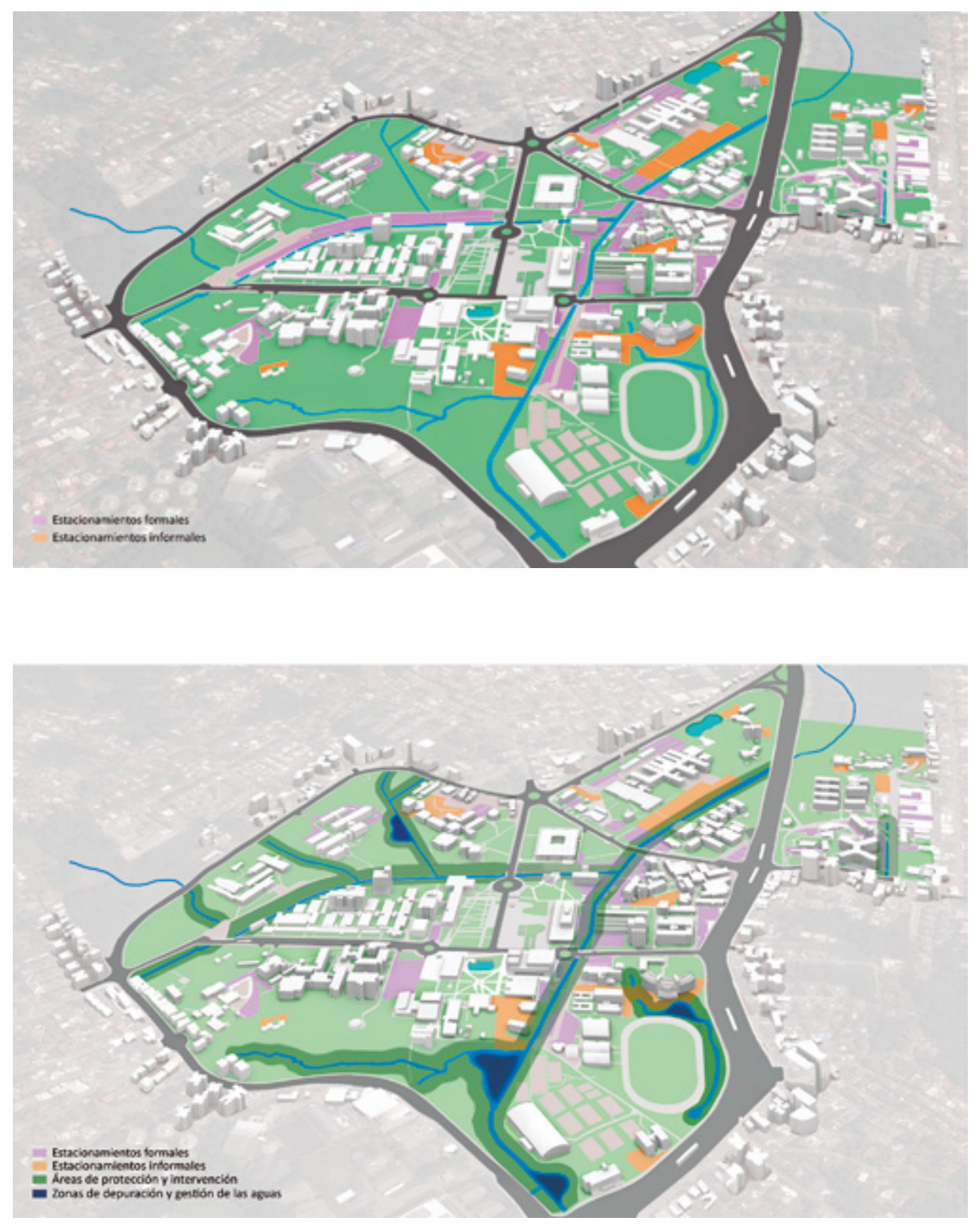

Figura 6 y 7 Áreas de intervención en el campus. Fuente: Laboratório de Ecologia Urbana (UFSC) 


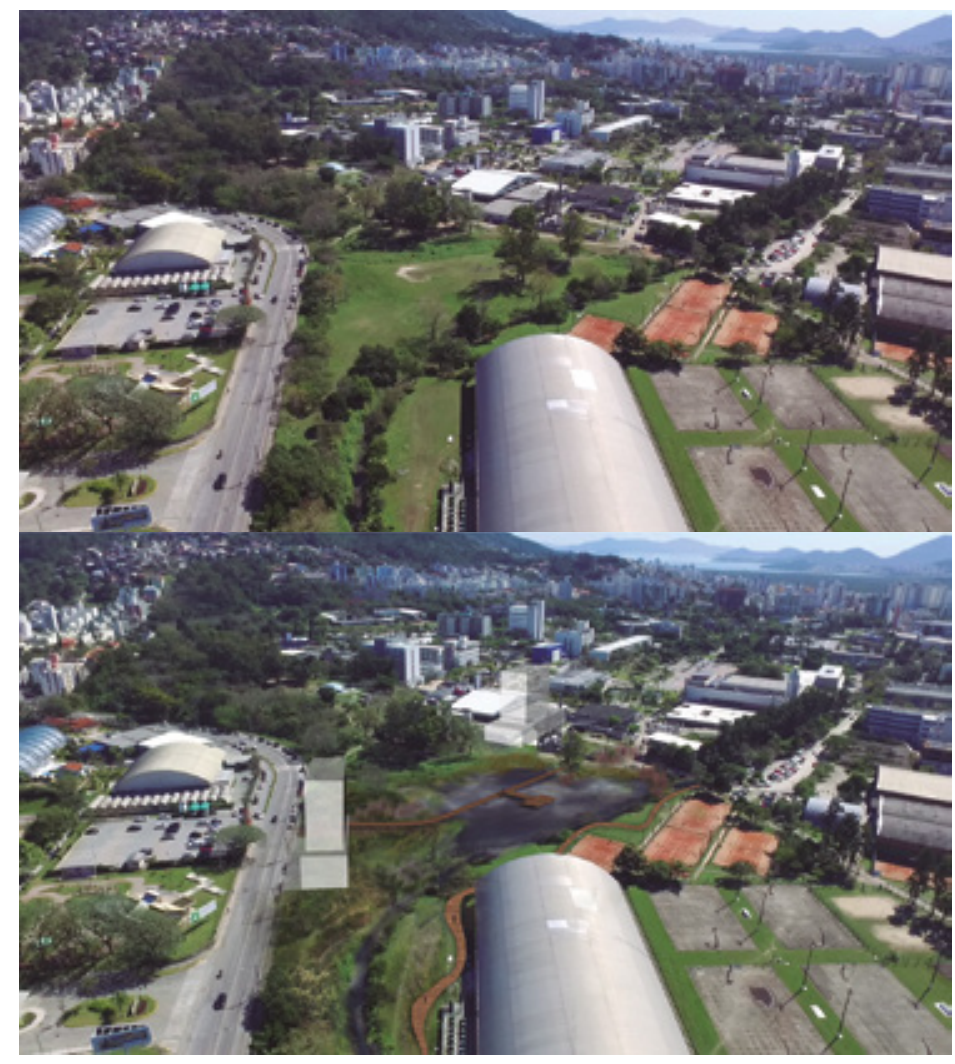

Figura 8 Propuesta para el área de depuración de las aguas que ingresan al campus. Fuente: Laboratório de Ecologia Urbana (UFSC). Fotografía de Felipe Finger

A través del estudio de patrones relacionados con el espacio físico, los estudiantes comprenden los procesos de cambios evitan proyectos que busquen retornar a un estado natural, "original". Los cursos de agua redirigidos en la época de la implantación de la universidad y el ciclo de las aguas por todo el campus constituyen elementos críticos de la región y, por ende, considerados ejes organizadores de las propuestas. Los estacionamientos en los márgenes de los ríos son elementos positivos por ser fácilmente modificables para la creación de parques y ampliación de las zonas de influencia de las aguas. El desafío está en revertir un proceso de negación de calidad de estos canales a partir del cual los edificios de la universidad fueron construidos dando la espalda a estas áreas. Consiguientemente, las propuestas revelan que nuevos edificios deben ser construidos volviéndose hacia esos espacios verdes, como también que los parques deben conformar zonas de purificación; que es necesario que las zonas de inundación sean capaces de absorber las aguas en épocas de muchas lluvias y que la apropiación del campus por la comunidad académica esté directamente vinculada a estos espacios (Figuras 6 y 7). Una de las razones del distanciamiento de los canales es la calidad del agua, la que ingresa en el campus con elevados grados de polución. Una de las principales estrategias planteadas ante esta situación, es la creación de zonas de baño naturales en las áreas más elevadas, cerca de los límites del campus, en los cuales ocurren procesos de depuración de materia orgánica y de retención de residuos sólidos (Figura 8). Además, en las áreas con mayor disponibilidad de espacio en los márgenes de los canales otras zonas de depuración del agua serían integradas a las áreas de parques. Ahora bien, se requiere negociar con el ayuntamiento para que este apoye financieramente dichas intervenciones, toda vez que es su responsabilidad garantizar la calidad del agua, cuyos cursos se hallan contaminados por lanzamientos irregulares de desagüe doméstico.

Estimular procesos colaborativos en estudiantes graduados de diferentes carreras es esencial y constituye un gran desafío. Así, por un lado, la forma de estructurar sus ideas y comunicarlas varía mucho y puede percibirse como un obstáculo para la colaboración. Por otro lado, esta diversidad es también la contribución más significativa para abordar un problema de esta naturaleza. Se hace evidente que la diversidad en la universidad no debe ser evitada, pero es necesario encontrar formas de estimular constantes intercambios entre estudiantes durante todo el proceso de aprendizaje en la universidad para que la innovación surja de estos procesos colaborativos. De esta forma, al final de cada carrera, los profesionales estarán preparados e interesados en la formación de equipos que trabajan en forma transdisciplinar. Una reflexión clave para enfrentar la actual crisis ambiental apunta a que si es necesario interferir en la dinámica de los procesos naturales, no puede hacerse de forma fragmentada. Estas dinámicas, estudiadas en una escala local, deben ser evaluadas también en escalas más amplias, buscando siempre conexiones positivas con los sistemas humanos.

Otra cuestión notable de este estudio fue la forma en que se buscó sensibilizar a personas con capacidad de decisión sobre los diferentes aspectos relacionados 
con las propuestas organizadas por los estudiantes. En efecto, los estudiantes trabajaron con videos cortos para facilitar la comunicación de sus propuestas, que fueron decisivos para encontrar apoyo a las ideas planteadas. Gracias a esta metodología, los estudiantes también pudieron debatir sobre distintos caminos para viabilizar sus proyectos, o parte de ellos, a través de la incorporación de importantes actores con poder de decisión.

Los alumnos discutieron formas de promover alteraciones con impacto en la forma de aprendizaje en el campus, partiendo de las relaciones entre la comunidad académica y la dinámica de las aguas. Estas propuestas de intervenciones se orientaron especialmente a alterar el flujo de automóviles y estacionamientos, y a garantizar que los cursos de agua sean los estructuradores de los espacios libres de la universidad. Estos cursos de agua pasan a ser explorados como importantes espacios de circulación, áreas de encuentros, puntos de interés visual, a partir de los nuevos edificios, y también como sitios destinados a la filtración de aguas a través de sistemas naturales de baño y de control de inundaciones. Al aproximar a la comunidad académica a dichos cursos de agua, valorizándolos por sus posibilidades positivas para calificar y garantizar la seguridad a los espacios libres, la universidad transmite un mensaje de que la vitalidad de sus canales promueve la calidad de aprendizaje.

\section{CONCLUSIONES}

A través de la promoción de nuevas formas de relacionarse con el medio ambiente, la universidad puede ver nuevamente como los diferentes departamentos de relacionan y la importancia de estimular sus conexiones para un pensamiento complejo e integral. Las dinámicas de la naturaleza apoyan la comprensión de que no existe una única respuesta para determinado problema y que cada pequeño problema trae consigo una complejidad de nuevos problemas y maneras de abordarlos. Según Wu y Wu, "es importante pensar en los procesos aparentemente opuestos, como cambio versus estabilidad, creatividad versus conservación y flexibilidad versus eficiencia, no como paradojas, sino como dialécticas que deben coexistir para alcanzar una síntesis de resiliencia urbana" (2013: 226). Garantizar y estimular la coexistencia de estos diálogos es extremadamente importante, aunque poco frecuente, en el medio académico.

El costo de una intervención de esta naturaleza en el campus es relativamente pequeño, sobre todo si se contempla su visibilidad y su impacto en la comunidad académica, tanto en el corto como en el largo plazo. Integrar las dinámicas de la academia y de la naturaleza constituye un excelente ejemplo de aprendizaje para toda la comunidad. El diseño urbano posee "un papel esencial, no solo en la producción de hábitats urbanos más seguros y saludables, sino en la gestión de tornar legibles y tangibles los sistemas que sustentan la vida y en los cambios de percepción de lo que es posible" (Spirn, 2014:567). En proyectos como este es fundamental que las principales decisiones de diseño sean claramente perceptibles por parte de los usuarios. Es esencial, en suma, que la apreciación estética de tales decisiones sea un instrumento para animar a la comunidad académica en la comprensión de las dinámicas de los ecosistemas, y que dicha comprensión esté directamente relacionada a una propuesta pedagógica general de la universidad a un liderazgo de procesos responsables e integrales en aras de desarrollar nuevas formas de bienestar en el planeta.

\section{REFERENCIAS BIBLIOGRÁFICAS}

7GROUP y REED, Bill. The Integrative Design Guide to Green Building: Redefining the Practice of Sustainability. Hoboken: John Wiley \& Sons, 2009.

BRUNDTLAND, Gro Harlem. Report of the World Commission on Environment and Development: Our Common Future. Oxford: Oxford Univ Press, 1987.

BUCKMINSTER FULLER, Richard. Operating Manual for Spaceship Earth. Carbondale: Southern Illinois University Press, 1969.

CHOI, Bernard C. K. y PARK, Anita W. P. Multidisciplinarity, interdisciplinarity and transdisciplinarity in health research, services, education and policy: 1. Definitions, objectives, and evidence of effectiveness. Clinical and investigative medicine, 2006, vol. 29, nº 6, pp. 351-364.

GARRETT, Elizabeth. Inauguration Address [en línea], 2015. [Consultado 12 agosto 2017]. Disponible en: http:// archive.president.cornell.edu/docs/garrett-address.pdf

HES, Dominique y du PLESSIS, Chrisna. Designing for Hope: Pathways to Regenerative Sustainability. New York: Routledge, 2015.

LEFF, Enrique. Epistemologia ambiental. São Paulo: Cortez, 2001.

MCDONOUGH, William y BRAUNGART, Michael. Cradle to cradle: remaking the way we make things. New York: North Point, 2002.

MORIN, Edgar y PAKMAN Marcelo. Introducción al pensamiento complejo. Barcelona: Gedisa, 1994.

ORR, David W. Earth in mind: On education, environment, and the human prospect. Washington: Island Press, 2004.

RODRIGUES, Icles. A UFSC na década de 1960: outras histórias... En: NECKEL, Roselane y KÜCHLER, Alita Diana C. (org.). UFSC 50 anos: trajetórias e desafios. Florianópolis: UFSC, 2010, pp. 17-35.

SPIRN, Anne Whiston. Ecological Urbanism: A Framework for the Design of Resilient Cities. En: NDUBISI, Forster O. (ed.). The ecological design and planning reader. Washington: Island Press, 2014, pp. 557-571.

WACKERNAGEL, Mathis y REES, William. Our Ecological Footprint: Reducing Human Impact on the Earth. Gabriola Island: New Society Publishers, 1996.

WU, Jianguo y WU, Tong. Ecological Resilience as a Foundation for Urban Design and Sustainability. En: PICKETT, Steward T.; CADENASSO, Mary L. y MCGRATH, Brian (eds.). Resilience in Ecology and Urban Design: Linking Theory and Practice for Sustainable Cities. New York: Springer, 2013, pp. 211-229. 